\title{
Cilia-associated Respiratory (CAR) Bacillus Infection in Adult Red Deer, Chamois, and Roe Deer
}

Raffaella Bergottini, ${ }^{1,4}$ Silvana Mattiello, ${ }^{2}$ Luca Crippa, ${ }^{3}$ and Eugenio Scanziani ${ }^{1}{ }^{1}$ Dipartimento di Patologia Animale, Igiene e Sanità Pubblica Veterinaria, Istituto di Anatomia Patologica e Patologia Aviare, University of Milan; ${ }^{2}$ Istituto di Zootecnia, University of Milan; ${ }^{3}$ Novuspharma, Bresso, Milan, Italy; ${ }^{4}$ Corresponding author (email: raffaella.bergottini@unimi.it)

ABSTRACT: Cilia-associated respiratory (CAR) bacillus is an unclassified bacterium that colonizes the ciliated epithelium of airways in laboratory rats, laboratory mice, and laboratory and conventionally reared rabbits, cattle, goats, and pigs. Data on the prevalence of CAR bacillus infection in wild animals are lacking. The present study demonstrated the occurrence of the organism in wild red deer (Cervus elaphus hippelaphus), chamois (Rupicapra rupicapra), and roe deer (Capreolus capreolus) from the Val Fontana in northern Italy. Prevalence ranged from $26 \%$ for red deer to $56 \%$ for chamois, with a statistically significant negative correlation between CAR bacilli infection and the presence of lymphoid follicles.

Key words: CAR, cilia-associated respiratory, chamois, deer, Italy, wild.

Cilia-associated respiratory (CAR) bacillus is an unclassified filamentous bacterium that colonizes the ciliated epithelium of the airways. The bacterium has the same approximate length and diameter as cilia and lies parallel to them, and is histologically detectable by silver staining techniques (Van Zwieten et al., 1980; Ganaway et al., 1985; Kurisu et al., 1990; Shoji-Darkye et al., 1991; Fernandez et al., 1996). Nevertheless, host cilia are not essential for bacterial colonization, and as demonstrated by electron microscopy, non-ciliated cells can be colonized (Matsushita, 1991).

Natural infection with CAR bacillus was first recognized in laboratory rats (Van Zwieten et al., 1980), and was then described in laboratory mice (Griffith et al., 1988; Matsushita et al., 1989), laboratory and conventionally reared rabbits (Waggie et al., 1987; Kurisu et al., 1990; Cundiff et al., 1995; Caniatti et al., 1998), cattle (Hastie et al., 1993; Pravettoni et al., 2001), goats (Fernandez et al., 1996), and finally pigs (Nietfeld et al., 1995).

CAR bacillus infection in rats and mice has been related to chronic respiratory dis- ease (CRD), which is characterized by chronic suppurative bronchitis and bronchiolitis, the filling of the bronchial lumen by mucopurulent exudate, and marked leukocytic infiltration in the lamina propria. Usually, CAR bacilli are present along the apices of the entire ciliated respiratory epithelium (Percy et al., 1993). The presence of CAR bacillus in rabbits has been correlated with hyperplasia of laryngeal, tracheal, and bronchial epithelia; loss of cilia; and bronchusassociated lymphoid tissue (BALT) hyperplasia. Colonization of bacilli in this species seems to be restricted to epithelial cells of the trachea, and to a lesser extent, to principal bronchi (Caniatti et al., 1998). In domestic ruminants, CAR bacilli infection is correlated with tracheal inflammatory lesions (Oros et al., 1997; Pravettoni et al., 2001). These bacilli are unlikely to be primary pathogens in pigs because animals experimentally infected with Mycoplasma-free isolates did not develop macroscopic or microscopic lesions (Nietfeld et al., 1999).

Very little is known about the taxonomy of CAR bacillus, and the term "CAR bacillus" is often used as if it represents a single species. However, there is molecular evidence that different bacteria, possibly of different genera, represent CAR bacilli in different mammalian hosts (Cundiff et al., 1995).

Data on the prevalence of CAR bacillus infection in wild animals are lacking. The organism was detected in the trachea of wild rats (MacKenzie et al., 1981; Brodgen et al., 1993), but whether the organism is transmissible to other wild animals is not known. In the present study, CAR bacillus infection is described in wild red deer (Cervus elaphus hippelaphus), chamois (Rupicapra rupicapra), and roe deer (Capreolus capreolus) from the Val Fontana in northern Italy ( $46^{\circ} 13^{\prime}$ latitude; $12^{\circ} 27^{\prime}$ longitude). 
Twenty-three red deer, three roe deer, and 30 chamois brought by hunters to the local reference center at the end of each hunting day during 2001 were examined. Biometric parameters (including weight and height) were collected for each animal, and body conditions were scored using three categories: 1) poor, 2) moderate, or 3) good. The ages of the animals were estimated using teeth and horn evaluations. Animals underwent a full necropsy. A $2 \mathrm{~cm}$ long tract of trachea was sampled from each animal and immediately fixed in $10 \%$ neutral buffered formalin. A $1 \mathrm{~cm}$ long sample from the dorsal part of the trachea and a similar sample from the ventral part were processed for histology. After paraffin embedding, $5 \mu \mathrm{m}$ thick histological sections were obtained and stained with hematoxylin and eosin (HE) and Warthin-Starry. Histological examination was carried out in a blind fashion without comparing HE with Warthin-Starry slides. The HE sections were evaluated for the presence of inflammatory lesions that were scored as $(-)$ when absent, as $(+)$ when mild, as $(++)$ when mild multifocal or severe focal, or as $(+++)$ when severe multifocal. Warthin-Starry stained sections were evaluated for the presence of CAR bacilli and severity of colonization. Sections were examined at $400 \times$ magnification and positive cases were scored as $(+)$ when few bacilli were detectable, as $(++)$ when their presence was moderate, and as $(+++)$ when they were numerous. The statistical analysis considered the following parameters: sex, age (younger or older than six months), presence and number of CAR bacilli, diffuse lymphocytic infiltration, follicular lymphocytic infiltration, granulocytic infiltration, squamous metaplasia, and body condition score. Chisquare test (SAS, 1985) was calculated to test for a relationship between presence of CAR bacilli and species, sex, and age of the animals. Spearman's rank correlation to test for a relationship between presence of CAR bacilli and histopathological find-

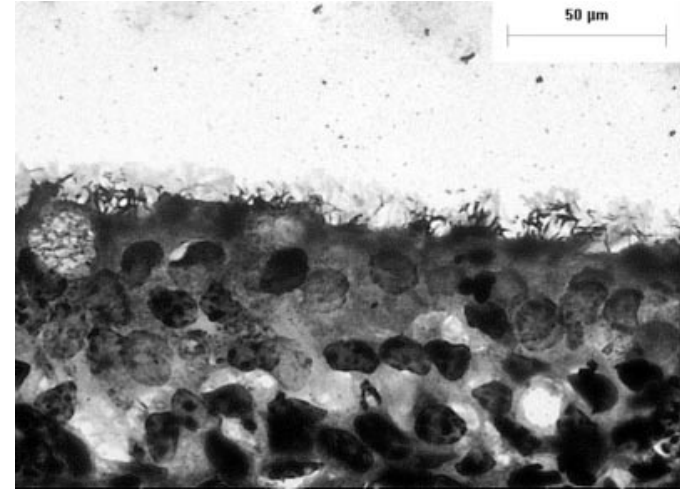

Figure 1. Tracheal mucosa from a deer (Cervus elaphus) colonized with filamentous CAR bacilli. Warthin-Starry. Bar $=50 \mu \mathrm{m}$.

ings was calculated and considered significant when $P<0.05$ (SAS, 1985).

Body conditions were good in 30 (53.5\%) animals, moderate in 19 (33.9\%), and poor in $7(12.5 \%)$. No significant respiratory or systemic lesions were detected at necropsy. CAR bacilli were histologically detected in the trachea of $24(42.9 \%)$ wild ruminants out of 56 examined: six out of 23 red deer $(26.1 \%)$, one out of three roe deer $(33 \%)$, and 17 out of 30 chamois $(56.7 \%)$. In Warthin-Starry stained sections, CAR bacilli appeared as blackstained, filamentous bacteria between the cilia of the respiratory epithelial cells (Fig. 1). CAR bacilli were few in 15 animals, moderate in number in eight animals, and numerous in one.

In HE stained sections, multifocal infiltrates of lymphocytes were often observed in the tracheal lamina propria. In 11 cases (19.6\%), lymphocytes were present as a diffuse infiltration: eight of them were scored as mild and three as mild multifocal or severe focal. In 16 cases (28.6\%), lymphocytic infiltrates were organized in follicular structures: 12 of them were scored as mild, three as mild multifocal or severe focal, and one as severe multifocal. In 13 cases $(23.2 \%)$, a diffuse infiltration of granulocytes, mainly neutrophils, was also detected. Granulocytic infiltration was mild in most cases; in two animals it was moderate, and in one the infiltration was se- 
vere, diffuse, and mainly composed of eosinophils. In this case, CAR bacilli were not observed and parasites or parasite-induced lesions of the respiratory tract were not observed at necropsy. Multifocal to diffuse squamous metaplasia of epithelial cells was present in 24 cases $(42.9 \%)$. Severe metaplasia was not observed, and the lesion was scored as mild in 12 animals and moderate in the remaining 12 . No ulcerative lesions were observed.

Although the Chi-square test demonstrated no correlation between sex, age, low body condition score, and presence of CAR bacilli, as well as between the presence of CAR bacilli and inflammatory lesions, a significant negative correlation between the severity of follicular lymphoid infiltrates and presence of CAR bacilli was found $(r=-0.28 ; \quad P<0.0334)$. Significant correlation was also found between the presence of diffuse lymphocytic infiltration and granulocytic infiltration $(r=0.29 ; P<0.281)$, and between squamous metaplasia and granulocytic infiltration $(r=0.54 ; P<0.0001)$.

The results of the present study demonstrate the presence of spontaneous CAR bacillus infection in wild red deer, roe deer, and chamois in the Val Fontana (Italy) with prevalence figures ranging from $26 \%$ for red deer to $56 \%$ for chamois. This prevalence is similar to that reported for domestic ruminants (Oros et al., 1997; Pravettoni et al., 2001). Prevalence observed in cattle ranged from $15.5 \%$ in veal to $37.7 \%$ in adult cattle (Pravettoni et al., 2001), whereas in goats it ranged from $21.2 \%$ in kids to $32 \%$ in adult goats (Oros et al., 1997). The only data available about prevalence of CAR bacillus in wild animals are $35 \%$ in a population of wild rats from central Iowa (Brodgen et al., 1993). Previous studies in numerous animal species and in domestic ruminants indicated that the tracheal mucosa represents the most frequent site for CAR bacilli colonization (Oros et al., 1997; Caniatti et al., 1998; Pravettoni et al., 2001). However, the fact that some animal species such as the ones considered in this study can display differ- ent localizations is a possibility that cannot be excluded. Further, the fact that a patchy distribution of the organisms on the tracheal mucosa might lead to an underestimation of positive animals cannot be excluded, either.

In this study, CAR bacillus was visualized directly on the Warthin-Starry stained histological sections, which represents the method of choice for the diagnosis of CAR bacillus infection. This is a routine diagnostic method that allows morphological identification of the organism in formalin fixed and paraffin embedded tissue. Other diagnostic techniques for the CAR bacillus identification include polymerase chain reaction (PCR) assay (Cundiff et al., 1994), and immunohistochemistry (Oros et al., 1996). Antibodies against different CAR bacilli are detectable using serological techniques, such as enzyme-linked immunoabsorbent assay (ELISA) or indirect immunofluorescent assay (Ganaway et al., 1985; Cundiff et al., 1995).

A culture for Mycoplasma spp. was not performed. It has been demonstrated that these organisms are primary pathogens and a common contaminant in CAR bacilli isolates from calves and pigs (Nietfeld et al., 1999). Furthermore, in a similar manner to CAR bacillus, mycoplasmas have been shown to localize between the cilia of the epithelial respiratory tract cells (Cheville, 1994). It is possible that they have a synergistic effect in damaging the cilia, and that concomitant infection or lack of infection with Mycoplasma spp. could have affected the result of this study.

Lymphocytic infiltration, granulocytic infiltration and squamous metaplasia were not correlated with the presence and number of CAR bacilli, indicating that these organisms are not the cause of inflammatory lesions. However, squamous metaplasia could have affected the ability to detect CAR bacilli. A noteworthy, statistically significant negative correlation between the presence of CAR bacillus and follicular lymphoid infiltration $(P<0.0334)$ was demonstrated. This finding might indicate the 
ability of the animal to clear CAR bacillus chronic infection via a specific immune response. All the data gathered in the present study indicate that CAR bacillus infection in wild ruminants does not induce significant morphologic alteration in the tracheal mucosa, with no or minimal influence on body conditions, which are indicative of the health state of the animals. Further investigation is required to determine whether the lack of disease associated with this CAR bacillus is due to low virulence of the bacteria or to significant host resistance.

\section{LITERATURE CITED}

Brodgen, K. A., R. C. Cutlip, and H. D. LehmKUHL. 1993. Cilia-associated respiratory bacillus in wild rats in central Iowa. Journal of Wildlife Disease 29: 123-126.

Caniatti, M., L. Crippa, A. M. Giusti, S. MattielLO, G. GRILli, R. Orsenigo, and E. SCANZIANI. 1998. Cilia-associated respiratory (CAR) bacillus infection in conventionally reared rabbits. Journal of Veterinary Medicine 45: 363-371.

Cheville, N. F. 1994. Ultrastructural pathology (An introduction to interpretation). Iowa State University Press, Ames, Iowa, 954 pp.

CundifF, D. D., C. Besch-Williford, R. R. HoOK, C. L. Franklin, and L. K. Riley. 1994. Detection of cilia-associated respiratory bacillus by PCR. Journal of Clinical Microbiology 36: 1930-1934.

$\longrightarrow,-,-$, $\longrightarrow$, and 1995. Characterization of cilia-associated respiratory bacillus isolates from rats and rabbits. Laboratory Animal Science 44: 22-26.

Fernandez, A., J. Oros, J. L. Rodriguez, J. King, AND J. B. POVEDA. 1996. Morphological evidence of a filamentous cilia-associated respiratory (CAR) bacillus in goats. Veterinary Pathology 33: 445-447.

Ganaway, J. R., T. H. Spencer, T. D. Moore, and A. M. ALLEN. 1985. Isolation, propagation and characterization of a newly recognized pathogen, cilia-associated respiratory bacillus of rats, an etiological agent of chronic respiratory disease. Infection and Immunity 47: 472-479.

Griffith, J., W. W. J. White, P. J. Danneman, and C. M. LANG. 1988. Cilia-associated respiratory (CAR) bacillus infection in obese mice. Veterinary Pathology 25: 72-76.

Hastie, A. T., L. P. Evans, AND A. M. Allen. 1993. Two types of bacteria adherent to bovine respiratory tract ciliated epithelium. Veterinary $\mathrm{Pa}-$ thology 30: 12-19.

Kurisu, K., S. Kyo, Y. Shimoto, and S. MatsushITA. 1990. Cilia-associated respiratory bacillus infection in rabbits. Laboratory Animal Science 40: 413-415.
MacKenzie, W. F., L. S. Magill, and M. Hulse. 1981. A filamentous bacterium associated with respiratory disease in wild rats. Veterinary $\mathrm{Pa}-$ thology 18: 836-839.

Matsushita, S. 1991. Ultrastructure of respiratory tract epithelium of rats experimentally infected with the CAR bacillus. Journal of Veterinary Medical Science 53: 361-363.

- T. Matsumoto, and K. FukUtsu. 1989. Transmission experiments of cilia-associated respiratory bacillus in mice, rabbits and guinea pigs. Laboratory Animals 23: 96-102.

Nietfeld, J. C., C. L. Franklin, L. K. Riley, D. H. Zeman, and B. T. GrofF. 1995. Colonization of the tracheal epithelium of pigs by filamentous bacteria resembling cilia-associated respiratory bacillus. Journal of Veterinary Diagnostic Investigation 7: 338-342.

, B. L. Fickbohm, D. G. Rogers, C. L. FrANKLIN, AND L. K. RILEY. 1999. Isolation of cilia-associated respiratory (CAR) bacillus from pigs and calves and experimental infection of gnotobiotic pigs and rodents. Journal of Veterinary Diagnostic Investigation 11: 252-258.

Oros, J., S. Matsushita, J. L. Rodriguez, F. RodRIGUEZ, AND A. FERnANDEZ. 1996. Demonstration of rat CAR bacillus using a labelled streptavidin biotin (LSAB) method. Journal of Veterinary Medical Science 58: 1219-1221.

- A. Fernandez, J. L. Rodriguez, C. L. Franklin, S. Matsushita, and J. B. Poveda. 1997. Association of cilia-associated respiratory (CAR) bacillus with natural chronic tracheitis in goats. Journal of Comparative Pathology 117(3): 289-294.

Percy, D. H., And S. W. Barthold. 1993. Rat. In Pathology of laboratory rodents and rabbits. Iowa State University Press, Ames, Iowa, 229 pp.

Pravettoni, D., S. Toccacieli, S. Monestiroli, L. Crippa, E. Canali, S. Salvi, and E. SCanziani. 2001. Cilia-associated respiratory (CAR) bacillus infection in veal calves and adult cattle. Deutsche tierarztliche Wochenschrift 108: 386-389.

SAS. 1985. SAS User's Guide Basics (version 5 edition). SAS Institute Inc., Cary, North Carolina, 956 pp.

Shoji-Darkye, Y., T. ITOH, AND N. KagiYama. 1991. Pathogenesis of CAR bacillus in rabbits, guinea pigs, syrian hamsters, and mice. Laboratory Animal Science 41: 567-571.

Van Zwieten, M. J., H. A. Solleveld, J. R. Lindsey, F. G. DE GRoOT, Zurcher, AND C. F. HollanDER. 1980. Respiratory disease in rats associated with a filamentous bacterium: A preliminary report. Laboratory Animal Science 30: 215-221.

Waggie, K. S., T. H. SPEnCER, and A. M. Allen. 1987. Cilia-associated respiratory (CAR) bacillus infection in New Zealand White rabbits. Laboratory Animal Science 37: 533

Received for publication 29 April 2004. 